\title{
Proposed new research ethics guidelines generate debate
}

$\mathrm{D}$ raft changes to the Declaration of Helsinki, which turns 50 next year, are causing controversy among bioethicists who monitor the global guide to conducting ethical research involving humans.

The World Medical Association (WMA) invites comments on the revisions (www.wma.net/en/20activities /10ethics/10helsinki/15publicconsult /index.html) from experts and stakeholders until June 15 via email to the WMA secretariat (doh@wma.net).

One of the contentious sections contains new wording concerning research involving vulnerable populations, such as children or individuals with mental illness. The 2008 version of the declaration allows for research on a vulnerable group if "the research is responsive to the health needs and priorities" of the group and members are likely to benefit from the research. The revised draft adds one more requirement: the research must also be a project that "cannot be carried out in a non-vulnerable population."

The new wording is potentially devastating for researchers and unjust to research participants, says Doris Schroeder, director of the Centre for Professional Ethics at the University of Central Lancashire in Preston, England. Schroeder believes, if adopted, the new wording could result in a ban on a decades-long study of Nairobi sex workers, some of whom remain free of AIDS despite years of unprotected sex. The women are impoverished and their work is illegal in Kenya, making them highly vulnerable. In establishing the research, scientists at the universities of Manitoba and Nairobi set up a clinic in the slum where the women live. The women have told interviewers they are grateful for access to quality health care and other benefits they've experienced as a result of the study.

If the draft revisions take effect, says Schroeder, these women could not participate in the study.

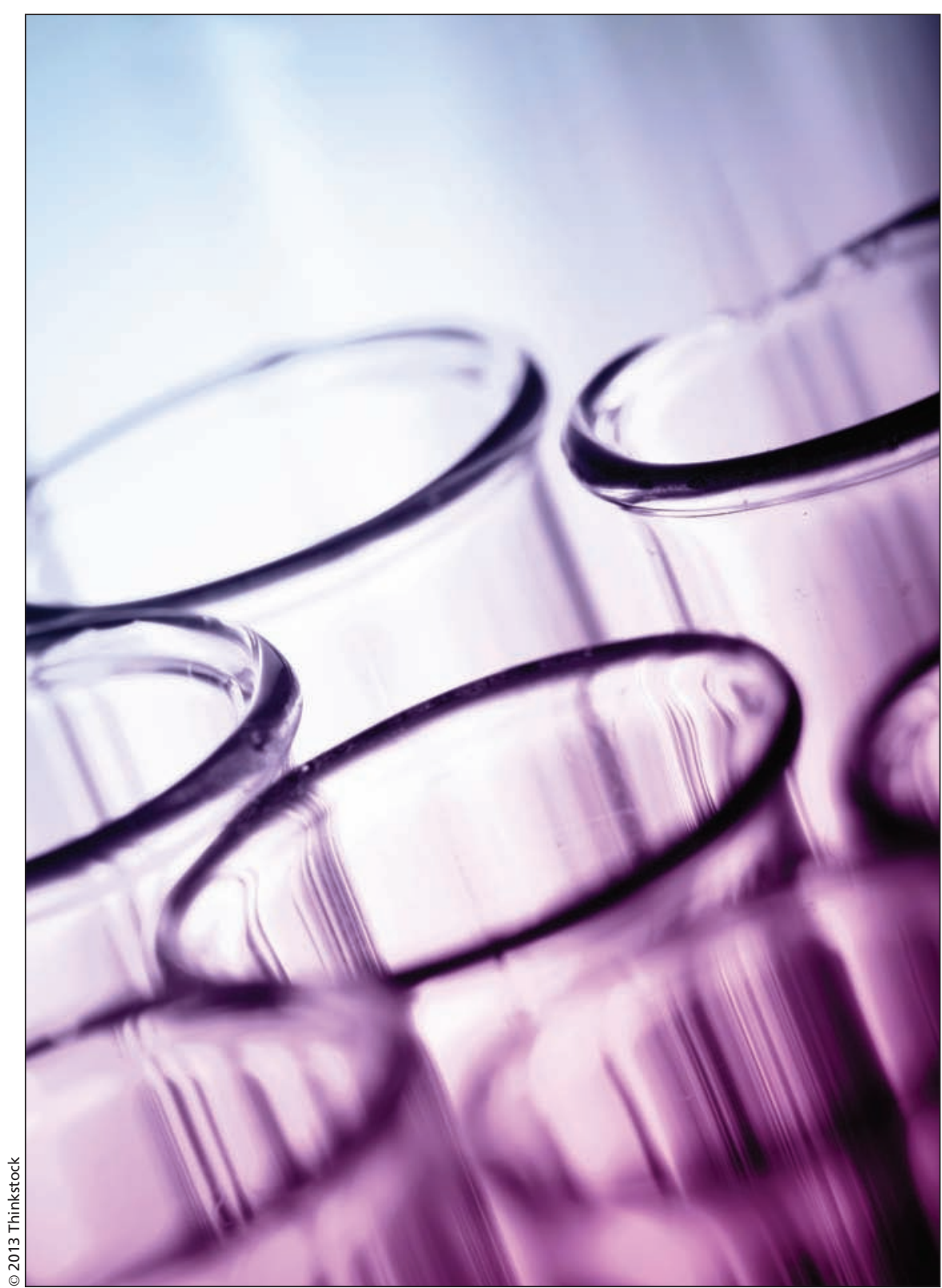

Experts and stakeholders are invited to comment on the draft revision of the Declaration of Helsinki, last updated in 2008.

"This research would have to be undertaken in a first-world country first," she says. "This is a protection mechanism that totally overshoots its target."

Jeff Blackmer, director of the Canadian Medical Association's Office of Ethics and an expert consultant to the WMA, interprets the language differently. "It's highlighting the fact that vulnerability has to be an important part of why you're doing the research involving that population or community," he explains.

Blackmer says he is pleased the revised draft encourages researchers or sponsors to provide poor or vulnerable communities with "a fair level of additional benefits," meaning sponsors 
could, for example, expand a local hospital or provide some other incentive for the community to participate in research.

"I'm hoping people will look at this and say, "That actually makes a lot of sense. You should expect benefits [from research] and you should also look for additional benefits," Blackmer says.

Another contentious change in the new draft concerns a requirement for access to study drugs after trials are complete. The 2008 version stated that patients in a study are entitled to "share any benefits that result from it, for example, access to interventions identified as beneficial in the study or to other appropriate care or benefits." The phrase was interpreted as entitling research participants to drugs they had received during the trial or to other benefits, to cover drugs still in development that would not become available for many years or for studies that did not test an intervention.

That wording was criticized as unclear, says Blackmer. The revised draft is more specific, requiring "posttrial access for all participants who still need an intervention identified as beneficial in the study."

But Schroeder says this narrows researchers' obligations. Only participants in clinical trials will have "posttrial access," not individuals enrolling in other studies, such as genetic studies.

Ethicists also anticipate extensive feedback concerning new wording around placebo-controlled trials. In 2008, the declaration allowed researchers to use placebo controls if they were considered scientifically necessary for the study and if patients receiving placebo would not experience "any risk of serious or irreversible harm."

Now, researchers will be allowed to use placebos as controls if patients will not experience "additional risks of serious or irreversible harm as a result of not receiving the best proven intervention."

This is "an overly permissive standard to hold trials to," says Jonathan Kimmelman, a bioethicist at McGill University, Montréal, Quebec, who has attended meetings of the WMA's Declaration of Helsinki Workgroup. He worries that, if adopted, the wording could allow researchers to leave patients with serious conditions untreated in order to test a new drug.

Doctors in African countries are also concerned about the placebo issue because they've already seen problems, says Dr. Margaret Mungherera, the Ugandan psychiatrist who is WMA president-elect. Mungherera has heard from colleagues who protested against pharmaceutical companies running trials without offering the best current treatment, she says.

"I was told there were circumstances where if it wasn't for people putting their foot down, they would have come in with placebo," she says. But the general consensus among people she speaks to is that the revised paragraph about placebo controls "is okay."

While developed countries such as Canada have their own codes of conduct for research, doctors in developing nations, "really depend on this document as their beacon," says Blackmer. That's why tiny changes in it matter so much. - Miriam Shuchman MD, Toronto, Ont.

CMAJ 2013. DOI:10.1503/cmaj.109-4498 